\title{
sciendo
}

\section{REGULATION OF FOLLICULOGENESIS BY GROWTH FACTORS IN PIGLET OVARY EXPOSED PRENATALLY TO $\beta$-HYDROXY- $\beta$-METHYLBUTYRATE (HMB)*}

\author{
Monika Hułas-Stasiak ${ }^{1 \bullet}$, Joanna Jakubowicz-Gil ${ }^{1}$, Piotr Dobrowolski ${ }^{1}$, Małgorzata Grzesiak ${ }^{2}$, \\ Siemowit Muszyński ${ }^{3}$, Małgorzata Świątkiewicz ${ }^{4}$, Ewa Tomaszewska ${ }^{5}$
}

\author{
'Department of Functional Anatomy and Cytobiology, Maria Curie-Sklodowska University, \\ Akademicka 19, 20-033 Lublin, Poland \\ ${ }^{2}$ Department of Endocrinology, Institute of Zoology and Biomedical Research, \\ Jagiellonian University in Krakow, Gronostajowa 9, 30-387 Kraków, Poland \\ ${ }^{3}$ Department of Biophysics, Faculty of Environmental Biology, University of Life Sciences in Lublin, \\ Akademicka 13, 20-950 Lublin, Poland \\ ${ }^{4}$ Department of Animal Nutrition and Feed Science, National Research Institute of Animal Production, \\ 32-083 Balice n. Kraków, Poland \\ ${ }^{5}$ Department of Animal Physiology, Faculty of Veterinary Medicine, University of Life Sciences \\ in Lublin, Akademicka 12, 20-950 Lublin, Poland \\ •Corresponding author: monhul@o2.pl
}

\begin{abstract}
$\beta$-hydroxy- $\beta$-methylbutyrate (HMB) is one of the leucine metabolites with protein anabolic effects which makes it very popular among athletes. Previously, it was shown that HMB administered during the prenatal period reduced the pool of primordial follicles and increased the proportion of developing follicles in newborn piglets. This work is a further step to understand these morphological alterations. Therefore, the aim of this study was to examine the effect of prenatal HMB treatment on the expression of the Kit ligand, BMP-4, bFGF, and the IGF-1/IGF-1R system which are the main growth factors controlling follicular development. Excised ovaries from 12 newborn piglets, originated from the control $(n=6)$ and HMB-treated $(n=6)$ sows were used for immunohistochemical and western-blot analysis. The tested proteins were localized within egg nests and ovarian follicles. Furthermore, the western-blot assay indicated higher BMP-4, Kit ligand, and IGF-1R expression, while the level of bFGF and IGF-1 proteins decreased after HMB dietary treatment. These findings show that HMB included into sow diet can modulate the expression of growth factors and thereby alter ovarian morphology in offspring. Therefore, this study opens a discussion about the benefits and risks of the diet supplemented with HMB and its potential application in medicine and animal husbandry, and further research is necessary in this area.
\end{abstract}

Key words: $\beta$-hydroxy- $\beta$-methylbutyrate, folliculogenesis, piglets, growth factors

*This research was funded by National Science Centre (NCN, Poland), grant number 2017/01/X/ NZ3/01055. 
In recent years, it has become very popular to use dietary supplements. One of them is $\beta$-hydroxy- $\beta$-methylbutyrate (HMB), which is a metabolite of leucine. HMB is considered an ergogenic substance that is a source of energy for muscles increasing their efficiency (Wilson et al., 2008, 2013). The studies on the effect of $\beta$-hydroxy- $\beta$-methylbutyrate on foetal development are of particular interest. HMB administered to pregnant females increased the birth weight of piglets (Blicharski et al., 2017; Tatara et al., 2007), improved their health condition (Flummer et al., 2012), and stimulated the development of the musculoskeletal system (Blicharski et al., 2017; Tatara et al., 2007; Wan et al., 2016 a, b, 2017). All these beneficial effects give hope for the potential use of HMB in prenatal medicine. HMB could be used in mothers at risk of premature delivery or to prevent IUGR (intrauterine foetal growth inhibition), which would accelerate the growth and maturation of the foetus and increase his chances of survival (Cieślak and Nieradka-Iwanicka, 2018). However, besides the huge number of positive HMB effects described in the literature, there are data reporting that prenatally administered HMB can exert a harmful effect on the foetus. HMB was found to increase insulin resistance in piglets, which in the future might predispose them to obesity and diabetes (Wan et al., 2016 a). Moreover, brain developmental disorders were observed in newborn males of the spiny mouse (Krawczyk et al., 2016).

It has been shown that many reproductive problems observed during puberty may be associated with developmental disorders in the prenatal and neonatal period (Chan et al., 2015; Evans et al., 2012; Dupont et al., 2012). Folliculogenesis is controlled not only by systemic endocrine hormones such as gonadotropins or steroids but also by a variety of intraovarian paracrine factors including growth factors and signaling molecules, which form a complex communication network in the ovarian follicles (Aaron et al., 2015; Morita and Tilly, 1999; Tingen et al., 2009). Through a series of activation steps, primordial follicles are transformed into primary follicles, and this process is critical for ovarian development (Tingen et al., 2009). The process of primordial follicle formation in pigs begins at day 56 of gestation and transition into the primary stage is first observed at day 90 of gestation (Bielańska-Osuchowska, 2006). Several growth factors appear to be important for follicle formation. The Kit ligand (also the SCF-stem cell factor), BMP-4 (bone morphogenetic protein 4), bFGF (basic fibroblastic factor), and the IGF-1/IGFR-1 system (insulin-like growth factor 1 and its cognate receptor) induce primordial follicle development and stimulate primordial to primary as well as primary to secondary follicle transition (Høyer et al., 2005; Morita and Tilly, 1999; Morita et al., 1999; Nilsson and Skinner, 2003, 2004).

Our previous findings indicated that prenatal HMB treatment dysregulated hormonal homeostasis and impaired early folliculogenesis, which led to ovarian morphology abnormalities in newborn piglets (Hułas-Stasiak et al., 2019). Therefore, we hypothesize that HMB may alter the expression of growth factors that regulate ovarian follicular development. To verify this hypothesis, the expression of the Kit ligand, BMP-4, bFGF, and the IGF-1/IGFR-1 system, which are involved in primordial follicle activation, was examined using Western-blot and immunohistochemistry in piglets originated from sows fed diet supplemented with HMB. 


\section{Material and methods}

The experiment was approved by the Local Ethics Committee on Animal Experimentation of the University of Life Sciences in Lublin, Poland (No. 2014/29).

\section{Animals and experimental design}

The study involved 12 newborn "before-first-colostrum-uptake" female piglets born by 12 sows (Puławska breed) after physiological farrowing and bred to the same boar. The control and experimental groups consisted of primiparous sows to avoid the effect of the number of pregnancies on the birth weight in the offspring. The pregnant sows at similar age (10 months) and with similar body weight (100-120 kg) were assigned into two equal groups: the control $(n=6)$ and experimental (HMBtreated, $n=6$ ). The animals were kept individually in standard conditions (controlled temperature, humidity, and 12:12h light/dark cycle). They were provided with free access to fresh water and fed twice a day $(2.3 \mathrm{~kg} /$ day $/ \mathrm{sow}$, at 08:00 a.m. and 04:00 p.m.) with well-balanced standard commercial diet formulated to meet or exceed the nutritional requirement for pregnant and lactating sows (NRC, 2012).

The experimental group were administered calcium salt of $\beta$-hydroxy- $\beta$ methylbutyrate (CaHMB; Lonza, Switzerland) at the dose of $0.2 \mathrm{~g} / \mathrm{kg}$ b.w./day between gestation days 70 and 90. The dose of HMB followed the report by Świetlicka and collaborators (Świetlicka et al., 2016). At a dose of $0.2 \mathrm{~g} / \mathrm{kg}$ b.w./day, HMB was found to exert a significant effect on enamel surface development in spiny mouse offspring. The days chosen for the HMB exposure are critical for ovarian development in pigs, i.e. the time of primordial follicle formation and the beginning of its transition into the primary stage. Powdered HMB was added to the first portion of food administered as the morning meal. The control group received placebo-supplemented feed $\left(\mathrm{CaCO}_{3}\right.$ at the daily dose of $0.2 \mathrm{~g} / \mathrm{kg}$ b.w.). Newborn female piglets from the control and HMB-treated groups were weighed (Table 1). Then only one female piglet was selected from each litter for the experiment with birth weight close to the average body weight of the other females in the litter (control, $n=6$; HMBtreated group, $n=6$ ). The detailed description of the birth weight and performance in newborn piglets in experimental groups is given in Table 1. The piglets were euthanized by intravenous injection of a lethal dose of pentobarbitalum natricum (Morbital, Biowet, Puławy, Poland) immediately after parturition. The ovaries were isolated and weighed.

Table 1. The effect of prenatal HMB supplementation on birth weight and performance in newborn piglets

\begin{tabular}{lcc}
\hline \multicolumn{1}{c}{ Parameter } & Control & HMB \\
\hline Birth weight of selected piglets $(\mathrm{g})$ & $880 \pm 85$ & $1230 \pm 122^{* *}$ \\
Birth weight of females in litter $(\mathrm{g})$ & $885 \pm 133$ & $1241 \pm 222^{* * *}$ \\
Litter size & $10.5 \pm 0.5$ & $9.5 \pm 1.2$ \\
Litter weight (kg) & $12.3 \pm 0.6$ & $14.7 \pm 0.9^{* *}$ \\
Number of females per litter & $5.33 \pm 0.5$ & $4.66 \pm 0.5^{*}$ \\
Sex ratio, female to male & $1.04 \pm 0.1$ & $0.95 \pm 0.2$ \\
\hline
\end{tabular}

${ }^{1}$ Data are presented as mean $\pm \mathrm{SD} ; * \mathrm{P}<0.05 ; * * \mathrm{P}<0.01 ; * * * \mathrm{P}<0.001$. 


\section{Histology}

The right ovaries from the newborn piglets (12 ovaries in total) were fixed in phosphate-buffered $4 \%$ paraformaldehyde for $24 \mathrm{~h}$ at room temperature, dehydrated, and embedded in Paraplast (Sigma-Aldrich, St. Louis, MO, USA). The ovaries were serially sectioned at $5 \mu \mathrm{m}$ thickness on a rotary microtome. Consecutive sections were placed on polysine-coated glass slides (SuperFrost Plus, Thermo Scientific, Germany) and processed for routine immunohistochemistry.

\section{Immunohistochemistry}

Briefly, the Paraplast sections were deparaffinised, rehydrated, and microwaved $3 \times 5 \mathrm{~min}$ in $10 \mathrm{mM}$ citrate buffer, $\mathrm{pH} 6.0$, to retrieve antigenicity. The activity of endogenous peroxidase was blocked with $3 \% \mathrm{H}_{2} \mathrm{O}_{2}$ in methanol $(1: 1)$ and nonspecific binding was prevented by application of the UltraCruz ${ }^{\circledR}$ Blocking Reagent (Santa Cruz Biotechnology, Santa Cruz, CA, USA). Rabbit polyclonal anti-Kit ligand, anti-BMP-4, anti-bFGF, anti-IGFR-1 (each in dilution 1:100, Abcam, Cambridge, UK) and anti-IGF-1 (1:100, LifeSpan BioSciences, Inc) antibodies were added to the tissue sections. Next, the samples were incubated overnight at $4{ }^{\circ} \mathrm{C}$. The antigens were visualized with the use of secondary biotinylated goat anti-rabbit IgG (1:200, $1 \mathrm{~h}$ at room temperature, Abcam, Cambridge, UK), an ImmunoCruz ${ }^{\circledR}$ ABC kit (30 min at room temperature; Santa Cruz Biotechnology, Santa Cruz, CA, USA), and a 3,3'-diaminobenzidine (DAB substrate kit, Abcam, Cambridge, UK), which is a substrate-staining chromogen. Tris-buffered saline (TBS) $\mathrm{pH} 7.6$ was used to rinse the sections after each step of the procedure. The sections were counterstained with Mayer's hematoxylin (Sigma-Aldrich, St. Louis, MO, USA). Next, the slides were dehydrated and mounted in DPX. The negative controls were not treated with the primary antibody. Finally, the slides were examined using an LSM5 Pascal microscope (Carl Zeiss, Jena, Germany) coupled with a digital camera (AxioCamHRc, Carl Zeiss, Jena, Germany) and a computer.

\section{Follicle classification}

The egg nests represented two subcategories based on their appearance: a healthy egg nest, i.e. a group of tightly packed oocytes surrounded by a layer of squamous follicular cells and egg nest breakdown with the loss of connections between degenerated oocytes and pre-granulosa cells migrating into the nests (Tingen et al., 2009). Follicles were identified following the classification system proposed by Pedersen and Peters (1968), which distinguishes primordial follicles with an oocyte surrounded by flattened granulosa cells, primary follicles with a single layer of cuboidal granulosa cells, and transitional follicles with 1-2 layers of columnar granulosa cells. Data on the number of individual types of egg nests and ovarian follicles were presented in a recent study (Hułas-Stasiak et al., 2019).

\section{Western-blot analysis}

The left ovaries obtained from the 6 experimental and 6 control piglets were used for western-blot analysis. The samples were homogenized in lysis buffer (125 mM TRIS-HCl pH 6.8; 4\% SDS; 10\% glycerol; 100 mM DTT), boiled in a water bath for 
$10 \mathrm{~min}$, and centrifuged at $10000 \times \mathrm{g}$ for $10 \mathrm{~min}$. Next, the supernatant was collected. The Bradford method (Bradford, 1976) was employed to determine the protein content. Samples containing $80 \mu \mathrm{g}$ of protein were separated by $10 \%$ SDS-PAGE and then electroblotted onto an Immobilon P membrane (Sigma-Aldrich, St. Louis, MO, USA). After the transfer, the membranes were blocked with 3\% low fat milk in PBS for $1 \mathrm{~h}$ and incubated overnight with polyclonal rabbit anti-Kit ligand, anti-BMP-4, anti-bFGF, anti-IGFR-1 (1:500, Abcam, Cambridge, UK) and IGF-1 (1:500, LifeSpan BioSciences, Inc) antibodies. The membranes were washed three times for 10 min with PBS containing 0.05\% TRITON X-100 (Sigma-Aldrich, St. Louis, MO, USA) and incubated for $2 \mathrm{~h}$ in the presence of a 1:30 000 dilution of alkaline phosphatase-conjugated goat anti-rabbit IgG (Abcam, Cambridge, UK). The membranes were visualized after addition of BCIP (5-bromo-4-chloro-3indolyl phosphate, Sigma-Aldrich) and NBT (nitrotetrazolium blue chloride, Sigma-Aldrich, St. Louis, MO, USA), which give a blue reaction colour. An anti- $\beta$-actin antibody (1:2000, Sigma-Aldrich) was used as the loading control. The blots were densitometrically quantified and normalized to their corresponding $\beta$-actin bands. In the end, the public domain ImageJ program with "Gel Analysis" function was used. Semi-quantitative analysis was performed for three separately repeated experiments from each control and HMB-treated group.

\section{Image analysis}

The slides were subjected to immunohistochemical assays in the same laboratory conditions to obtain comparable staining intensity. The next step consisted in quantitative estimation of the intensity of the immunohistochemistry reaction (IHC) for the following proteins: Kit ligand, BMP-4, bFGF, IGF-1, and IGFR-1. This was achieved by analysis of digital images from at least 6 sections (for each proteins) of ovaries from each control and HMB-treated female. In total, 36 sections (per group for each protein) were estimated. The digital colour images were registered with the use of an LSM 5 Pascal confocal microscope (Carl Zeiss, Jena, Germany) equipped with an AxioCamHRc camera (Carl Zeiss, Jena, Germany) and coupled with a computer. A 40x objective was used to capture images of egg nests and primordial, primary, and transitional ovarian follicles. All images were saved as 24-bit RGB colour images in TIFF (Tagged Image File Format) for quantification. A colour threshold function of ImageJ (Wayne Rasband, National Institute of Mental Health, Bethesda, MD, USA) was used to separate DAB stained pixels from the background. The hue, saturation, and brightness for a randomly chosen image were established to define the colour threshold range for DAB. Then, the selected threshold was applied to extract the positively stained colour pixels (DAB) from the background (hematoxylin stained pixels) in the analysed images. Ten separate measurements (per section) for each type of the egg nest and ovarian follicles were made. The intensity of the IHC reaction was evaluated in the 8-bit greyscale. The pixel intensity values in the digital image analysis range from 0 to 255 , with 0 representing the black and 255 the white shade of grey. For better illustration of the results (graphs in the text), the pixel brightness values were measured on inverted images in the 8-bit greyscale (from 0 -white to 255-black). 


\section{Statistical analysis}

All results are expressed as means $\pm \mathrm{SD}$. Normal distribution of data was checked with the Shapiro-Wilk test. Brown-Forsythe tests were applied to determine the equality of variance. Data were analysed with the use of Mann-Whitney U test when normal distribution of data and/or equality of variance were not met. A P-value less than 0.05 was considered statistically significant. All statistical analyses were carried out using STATISTICA 13.1 software (StatSoft, Inc., 2017).

\section{Results}

\section{Immunohistochemistry}

\section{BMP-4 immunolocalization}

In the investigated ovaries from the control and HMB-treated piglets, BMP-4 staining was detected within egg nests and follicles (Figure $1 \mathrm{~A}-\mathrm{D}$ ). However, BMP4 staining observed in the oocytes within healthy and breakdown egg nests increased significantly $(\mathrm{P}<0.001, \mathrm{P}<0.01$, respectively $)$ in the HMB-treated group in comparison with the control (Figure $1 \mathrm{E}$ ). Interestingly, in the control group, BMP-4 labelling was mainly limited to the granulosa cells of primordial follicles (Figure 1 D inset), while in the HMB-treated group oocytes were also stained positively (Figure $1 \mathrm{~B}$ inset). In turn, in the more advanced follicular stages (primary and transitional), the BMP-4 protein was predominantly localized in the granulosa cells; however, diffuse staining was also noted in the oocytes in both examined groups of piglets (Figure $1 \mathrm{~B}, \mathrm{D}$ ). Following the HMB treatment, the intensity of the BMP-4 immunostaining increased significantly in the primordial $(\mathrm{P}<0.01)$, primary $(\mathrm{P}<0.01)$, and transitional $(\mathrm{P}<0.05)$ follicles compared with the control group (Figure $1 \mathrm{E})$.

\section{Kit ligand}

Kit ligand staining was observed in the oocytes present within the healthy and breakdown egg nests in the HMB-treated and control groups (Figure 2 A, B, F). The HMB treatment resulted in significantly increased immunostaining within healthy egg nests $(\mathrm{P}<0.05)$, compared with the control group (Figure $2 \mathrm{H}$ ). There were no statistical differences in the breakdown egg nests in this regard between the two examined groups (Figure $2 \mathrm{H}$ ). However, in the HMB-treated group, the intensity of Kit ligand staining in the breakdown egg nests was reduced $(\mathrm{P}<0.05)$, compared with the healthy nests (Figure $2 \mathrm{H}$ ). Furthermore, the Kit ligand protein was also detected in the cytoplasm of the oocytes and granulosa cells of the primordial follicles in the HMB-treated group (Figure 2 D). However, in the control group the reaction was predominantly observed in granulosa cells of these follicles (Figure $2 \mathrm{E}$ ). The intensity of the Kit ligand immunostaining in the primordial, primary, and transitional follicles was significantly higher $(\mathrm{P}<0.001$, $\mathrm{P}<0.01, \mathrm{P}<0.01$, respectively) in the HMB-treated group than in the control (Figure $2 \mathrm{C}, \mathrm{G}, \mathrm{H})$. 

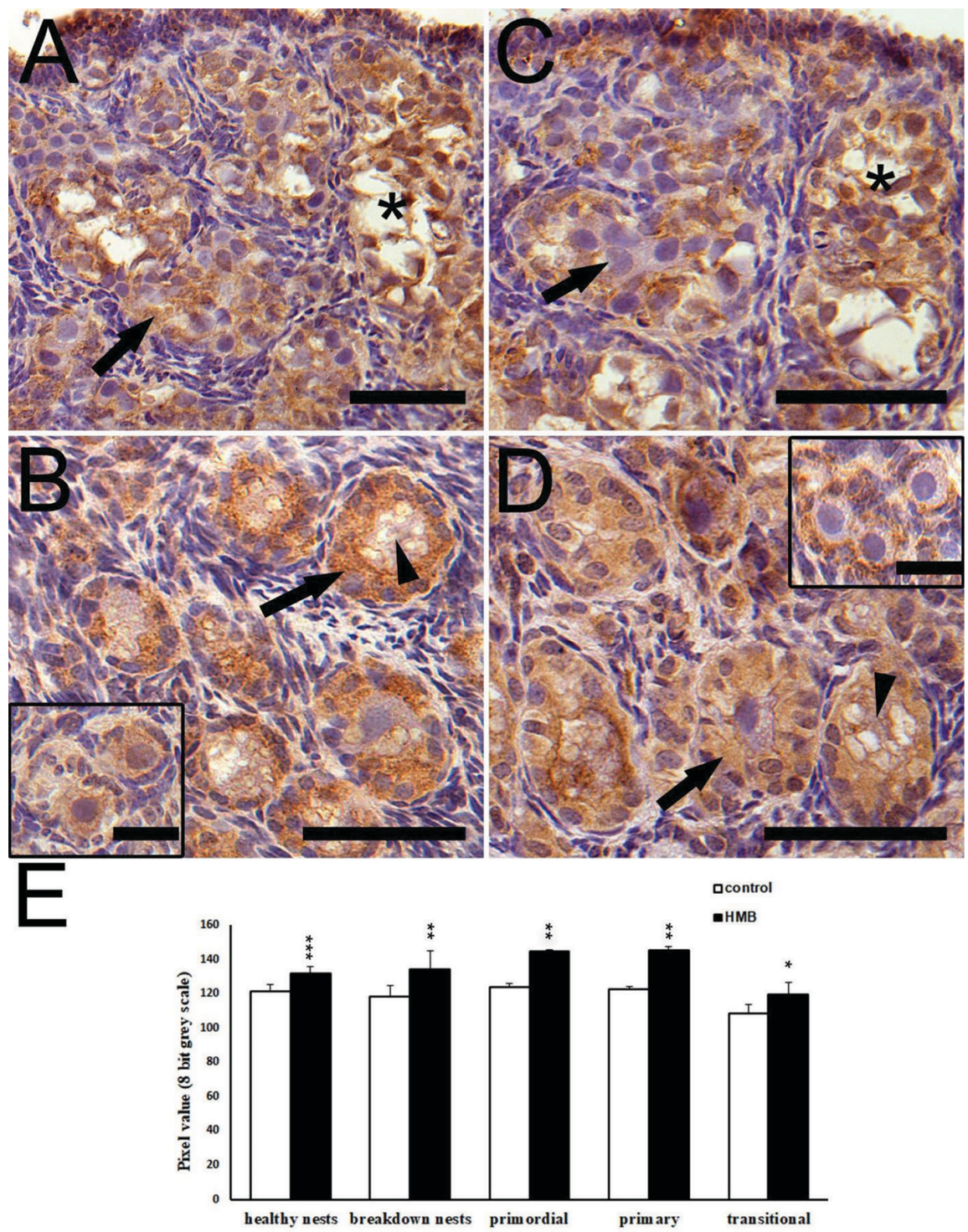

Data are presented as mean $\pm \mathrm{SD} ; * \mathrm{P}<0.05 ; * * \mathrm{P}<0.01 ; * * * \mathrm{P}<0.001$.

Figure 1. Immunolocalization of BMP-4 in HMB-treated (A, B) and control (C, D) ovaries from newborn piglets. Brown staining indicates positive reaction in the cells. BMP-4 was localized in oocytes within healthy (arrow) and breakdown (star) egg nest in both examined groups of piglets (A, C). In the HMB-treated group, oocytes and granulosa cells of primordial follicles were positively stained (B, inset), whereas in the control group BMP-4 immunostaining was mainly detected in granulosa cells (D, inset). In developing follicles, the BMP-4 protein was predominantly localized in granulosa cells (arrows); however, faintly stained oocytes (arrowheads) were also noted in both examined groups (B, D). Scale bars represent $100 \mu \mathrm{m}$. E. Chart represents the intensity of BMP-4 immunostaining in control and HMB-treated ovaries from newborn piglets 

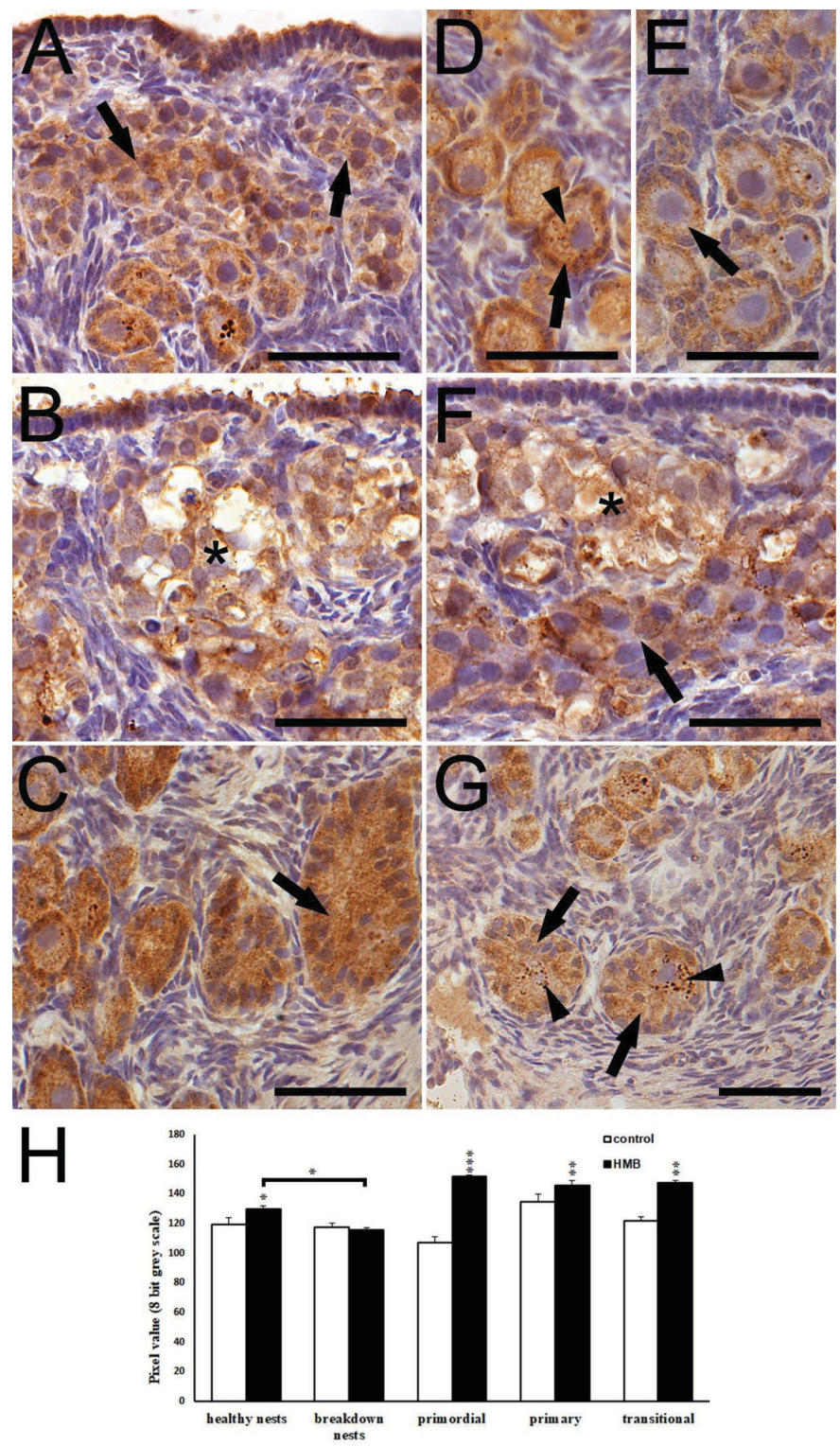

Data are presented as mean $\pm \mathrm{SD} ; * \mathrm{P}<0.05 ; * * \mathrm{P}<0.01 ; * * * \mathrm{P}<0.001$

Figure 2. Immunolocalization of Kit ligand in HMB-treated (A-D) and control (E-G) ovaries from newborn piglets. Kit ligand staining was detected within the healthy (arrows) and breakdown (stars) egg nests in the HMB-treated (A, B) and the control group (F). In the HMB-treated group, positive labelling was also observed in oocytes (arrowhead) and granulosa cells (arrows) of primordial follicles (D), while in the control group it was mainly localized in granulosa cells (E). Kit ligand staining was noted in oocytes (arrowhead) and granulosa cells (arrows) of developing follicles in both the HMB-treated (C) and the control group (G). Scale bars represent $100 \mu \mathrm{m}$. H. Chart represents the intensity of Kit ligand immunostaining in control and HMB-treated ovaries from newborn piglets 

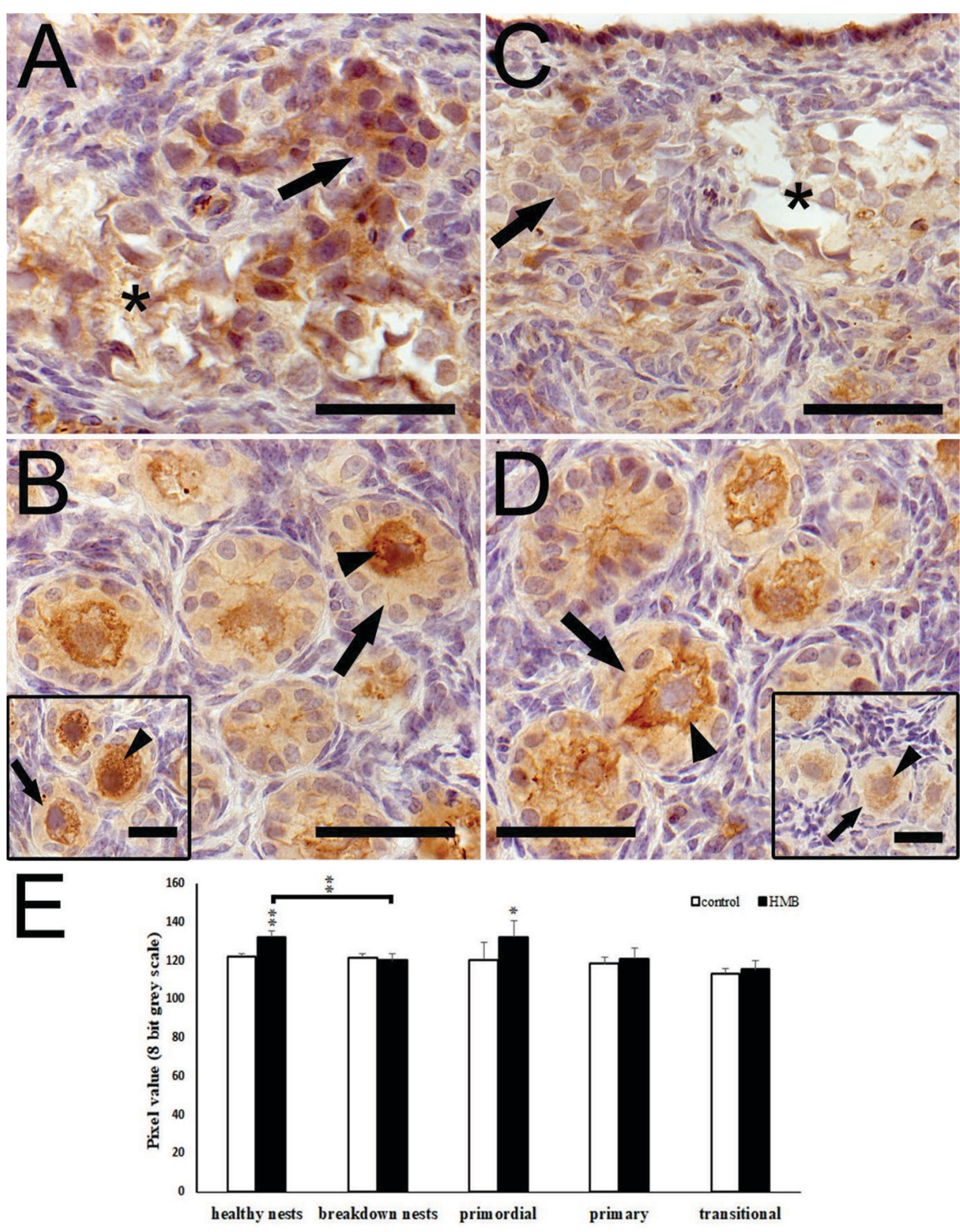

Data are presented as mean $\pm \mathrm{SD} ; * \mathrm{P}<0.05 ; * * \mathrm{P}<0.01$

Figure 3. Immunolocalization of IGFR-1 in HMB-treated (A, B) and control (C, D) ovaries from newborn piglets. Positive IGFR-1 labelling was noted in oocytes clustered within the healthy (arrows) and breakdown (stars) egg nests in the HMB-treated (A) and control group (C). Stronger immunostaining was observed in oocytes (arrowhead) while weaker in granulosa cells (arrows) of developing follicles in both examined groups (B, D). Furthermore, oocytes (arrowhead) from primordial follicles indicated more intensive immunosignal in the HMB-treated group (B, inset) than in the control (D, inset). Scale bars represent $100 \mu \mathrm{m}$. E. Chart represents the intensity of IGFR-1 immunostaining in control and HMB-treated ovaries from newborn piglets 

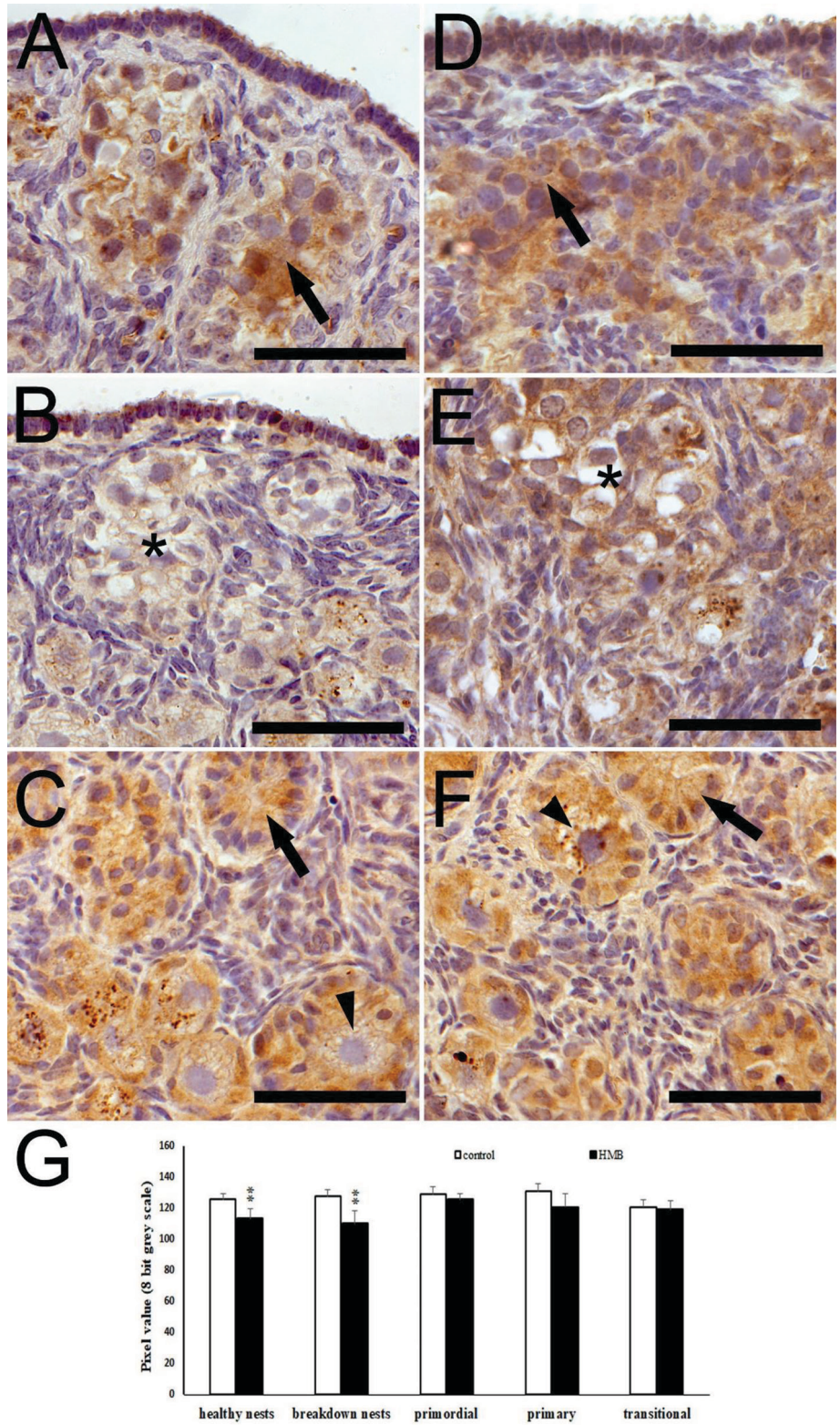

Data are presented as mean $\pm \mathrm{SD} ; * * \mathrm{P}<0.01$

Figure 4. Immunolocalization of IGF-1 in HMB-treated (A-C) and control (D-F) ovaries from newborn piglets. IGF-1 staining was noted in the healthy (arrows) and breakdown (stars) egg nests in the HMB-treated (A, B) and control group (D, E). In ovarian follicles, immunoreaction was predominantly seen in granulosa cells (arrows), while oocytes (arrowheads) were weakly labelled (C, F). Scale bars represent $100 \mu \mathrm{m}$. G. Chart represents the intensity of IGF-1 immunostaining in control and HMB-treated ovaries from newborn piglets 

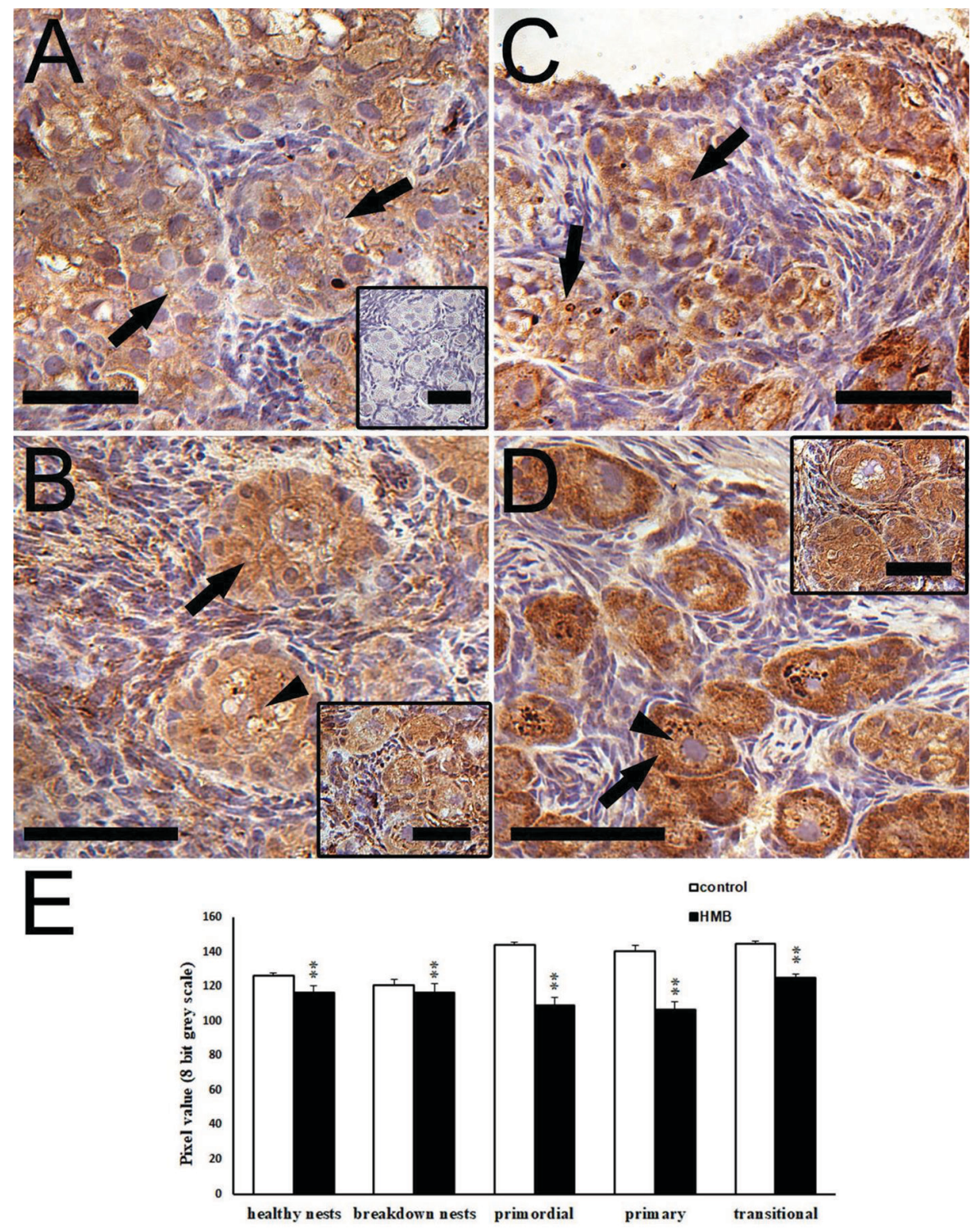

Data are presented as mean $\pm \mathrm{SD} ; * * \mathrm{P}<0.01$

Figure 5. Immunolocalization of bFGF in HMB-treated (A, B) and control (C, D) ovaries from newborn piglets. Positive bFGF immunostaining was visible in oocyte egg nests (arrows) in the HMB-treated (A) and control group (C). Oocytes (arrowheads) and granulosa cells (arrows) from all follicular stages indicated bFGF immunosignal in both the HMB-treated (B, B inset) and control group (D, D inset). A negative control was performed in the absence of primary antibodies (A, inset). Scale bars represent $100 \mu \mathrm{m}$. E. Chart represents the intensity of bFGF immunostaining in control and HMB-treated ovaries 


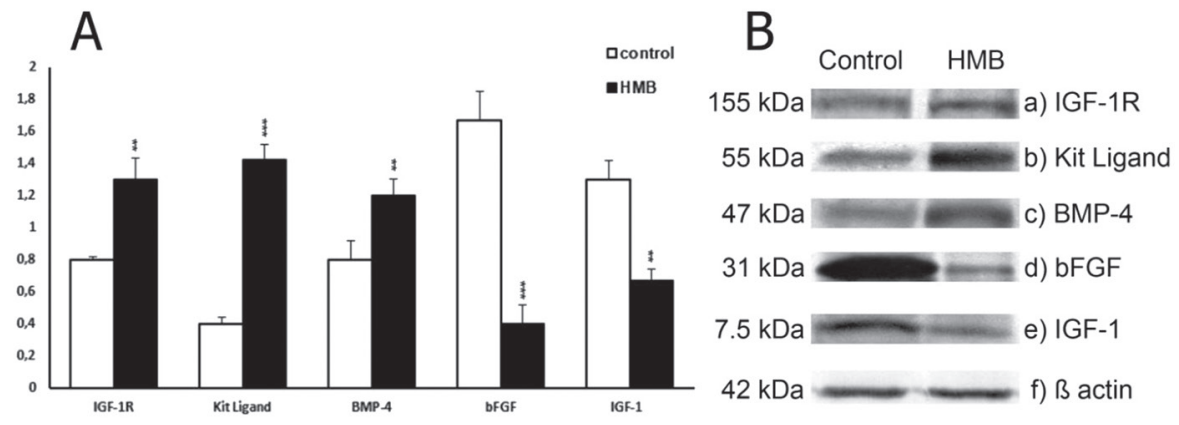

Data are presented as mean $\pm \mathrm{SD} ; * * \mathrm{P}<0.01 ; * * * \mathrm{P}<0.001$

Figure 6. Protein expression in the ovaries obtained from control and HMB-treated newborn piglets. (A) The relative abundance of IGF-1R, Kit ligand, BMP-4, bFGF, and IGF-1 was evaluated densitometrically and expressed as the ratio relative to $\beta$-actin.

(B) Representative Western blots of examined proteins in the ovaries obtained from the control and the HMB-treated group

\section{IGFR-1 and IGF-1 immunolocalization}

More intensive IGFR-1 labelling $(\mathrm{P}<0.01)$ was noted in the oocytes clustered within the healthy egg nests in the HMB-treated group than in the control (Figure 3 $\mathrm{A}, \mathrm{B}, \mathrm{C})$. There were no statistical differences in the breakdown egg nests between the experimental group and the control in this regard. However, as with the Kit ligand, the intensity of the IGFR-1 immunoreaction within the breakdown egg nests significantly decreased $(\mathrm{P}<0.01)$ compared to the healthy nests in the same HMBtreated group (Figure $3 \mathrm{E}$ ). Moreover, stronger immunostaining in the oocytes and weaker in the granulosa cells was observed in primordial and developing follicles in both groups examined (Figure $3 \mathrm{~B}, \mathrm{D}$ ). However, the IGFR-1 immunosignal in primordial follicles was significantly higher $(\mathrm{P}<0.05)$ in the HMB-treated group than in the control (Figure $3 \mathrm{~B}$ inset, D inset, E). There were no statistical differences between the primary and transitional follicles in this regard (Figure $3 \mathrm{E}$ ).

In contrast to IGFR-1, significantly weaker staining for IGF-1 was noted in the healthy and breakdown egg nests $(\mathrm{P}<0.01)$ in the HMB-treated group than in the control one (Figure 4 A, B, D, E, G). There were no statistical differences between the primordial, primary, and transitional follicles in this regard (Figure $4 \mathrm{G}$ ). The presence of the IGF-1 protein in the ovarian follicles was predominantly evident in the granulosa cells, whereas the oocytes were weakly labelled (Figure 4 C, F).

\section{$b F G F$}

Positive labelling was observed in the oocytes within the healthy and breakdown egg nests, in the oocytes and granulosa cells of the primordial and developing follicles in both the control and the HMB-treated group (Figure 5). Interestingly, the intensity of bFGF immunostaining in the egg nests as well as in all follicular stages decreased dramatically $(\mathrm{P}<0.01)$ in the HMB-treated group in comparison with the control (Figure $5 \mathrm{E}$ ). 


\section{Western-blot analysis}

Analysed proteins were observed as single bands at approximately $155 \mathrm{kDa}$ for IGF-1R, $55 \mathrm{kDa}$ for Kit ligand, $47 \mathrm{kDa}$ for BMP4, $31 \mathrm{kDa}$ for bFGF and $7.5 \mathrm{kDa}$ for IGF-1 (Figure $6 \mathrm{~B}$ ). In the ovaries of newborn piglets, the prenatal HMB treatment increased the expression of BMP4 $(\mathrm{P}<0.01)$, Kit ligand $(\mathrm{P}<0.001)$, and IGFR-1 $(\mathrm{P}<0.01)$, whereas the IGF-1 and bFGF protein levels were significantly decreased $(\mathrm{P}<0.01, \mathrm{P}<0.001$ respectively) in comparison with the control group (Figure $6 \mathrm{~A}$, B). The obtained results confirmed the immunohistochemical analysis.

\section{Discussion}

\section{Germ cell apoptosis}

There is a general agreement that only somatic components of the ovary produce the Kit ligand and IGF-1 growth factors, which have paracrine action on germ cell expressing the c-kit and IGFR-1 receptors (Behl and Kaul, 2002; Parrot and Skinner, 1999). However, in the present study, staining for the Kit ligand and IGF-1 proteins was also detected in oocytes clustered within egg nests of the porcine ovary. Indeed, there are studies which have confirmed the mRNA and protein expression of Kit ligand and its receptor c-kit in oogonia of the foetal mouse ovary (Doneda et al., 2002; Kang et al. 2003). Similar to our observations, the Kit ligand/c-kit as well as IGF-1/ IGFR-1 co-localization was documented in the human foetal germ cells (Høyer et al., 2005; Poljicanin et al., 2015).

Oogonia/oocytes survival depends on growth factors, and a limited supply of growth factors might determine their death (Hussein, 2005; Morita et al., 1999; Nilsson et al., 2002). An anti-apoptotic effect of the Kit ligand on primordial germ cells, oogonia, and oocytes has been demonstrated in foetal gonads (Driancourt et al., 2000). Our previous findings showed that the prenatally administered HMB increased the percentage of apoptotic oocytes within breakdown egg nests compared to the control (Hułas-Stasiak et al., 2019). Current findings indicated that the intensity of the Kit ligand immunostaining within healthy egg nests increased after the HMB administration; however, it was reduced during egg nest breakdown. This may therefore indicate the involvement of Kit ligand in the regulation of oocyte apoptosis into egg nests. Interestingly, Kit ligand alone has been shown to be completely ineffective in preventing oogonium and oocyte loss while a combination of survival factors is necessary to fully suppress germ cell apoptosis in an in vitro model (Morita and Tilly, 1999; Morita et al., 1999). The inclusion of IGF-1 alone into the culture medium significantly reduced germ cell apoptosis. However, the addition of all factors (IGF-1, Kit ligand plus LIF) to cultured ovaries maintained the germ cell numbers to levels that were not significantly different from those in the IGF-1-treated ovaries (Morita et al., 1999). It seems that IGF-1 alone is a potent survival factor and the main inhibitor of germ cell apoptosis in cultured foetal ovaries. In the present study the intensity of IGF-1 staining decreased in both healthy and breakdown egg nests in the HMB-treated group. Surprisingly, the intensity of IGF-1 receptor (IGFR-1) staining was significantly higher in healthy egg nests than in the breakdown egg 
nests. Thus, the altered IGF-1/IGFR-1 signalling and an insufficient level of IGF-1 and Kit ligand proteins may promote germ cell apoptosis within egg nests in the HMB-treated ovaries.

There are data reporting that another factor, i.e. BMP-4, can reduce the number of primordial germ cells (PGCs) in the human foetal ovary by promoting PGC apoptosis (Childs et al., 2010). Moreover, a culture of foetal mouse ovaries with BMP-4 has been shown to induce a testicular-like phenotype and reduce germ cell numbers (Ross et al., 2003).

Interestingly, after the HMB treatment, the intensity of BMP-4 staining increased significantly in the oocytes within the healthy and breakdown egg nests. Therefore, it cannot be excluded that BMP-4 is involved in germ cell death and reduction of the oocyte pool after HMB supplementation. Similarly, basic FGF signalling is also critical to proliferation of primordial germ cells thereby controlling the number of germ cells in mice (Resnick et al., 1998). In the present study, bFGF was localized in oocytes clustered within egg nests and the intensity in this compartment decreased in the HMB-treated group compared with the control. Moreover, the bFGF protein expression in the whole ovarian homogenates was dramatically lower after the HMB treatment. Therefore, an anti-apoptotic action of bFGF should not be excluded either.

The network of interactions between all these growth factors is needed to regulate nest breakdown and primordial follicle assembly (Tingen et al., 2009). The disruption of these intracellular signalling pathways may lead to enhanced germ cell apoptosis within egg nests. Our findings suggest that HMB may alter the expression of growth factors that cause oocyte apoptosis within egg nests.

\section{Follicle recruitment}

Our previous findings reported that HMB can accelerate follicular recruitment (Hułas-Stasiak et al., 2019). Therefore, we hypothesized that some of the growth factors mentioned above are involved in follicle recruitment and growth. One of them is the Kit ligand. Parrott and Skinner (1999) reported that the Kit ligand is predominantly produced in granulosa cells and acts as a signal from granulosa cells to oocytes and surrounding stroma to promote the primordial to primary follicle transition. However, its cognitive receptor c-kit is expressed in oocyte oolemma (Driancourt et al., 2000). Indeed, in the present study, Kit ligand staining was mainly observed in the granulosa cells of primordial and growing follicles in both examined groups. Surprisingly, in the HMB-treated group, the cytoplasm of oocytes from all follicles showed intense immunolabelling for the Kit ligand, in contrast to the control group, in which oocytes were faintly stained. Similar to our observations, the Kit ligand was also detected in the oocytes of primordial and growing follicles of neonatal mouse (Kang et al., 2003) and human ovaries (Høyer et al., 2005). It is very likely that the detection of the Kit ligand in the oocyte is a consequence of receptor-mediated endocytosis, and the Kit ligand protein but not mRNA can be immunolocalized within oocytes at all stages of folliculogenesis (Hut et al., 2006).

There are data reporting that the Kit ligand alone significantly increased the proportion of developing follicles in cultured neonatal rat ovaries (Nilsson and Skinner, 2003; Parrot and Skinner, 1999) and inhibited oocyte apoptosis in primordial fol- 
licles (Jin et al., 2004) as well as promoted granulosa cell proliferation of cultured mouse ovaries (Reynaud et al., 2000).

Similar to the Kit ligand, BMP-4 was attached to the growing list of paracrine growth factors that have been shown to regulate the primordial to primary follicle transition (da Cunha et al., 2017; Nilsson and Skinner, 2003; Shimizu et al., 2004). In the current study, BMP-4 staining was detected in the oocytes and granulosa cells of primordial and developing follicles in the HMB-treated group. However, in the control group, the BMP-4 labelling was mainly limited to granulosa cells of primordial follicles. This suggests that BMP-4 can be involved in the activation of oocytes in primordial follicles from HMB-treated piglets. Nilsson and Skinner (2003) observed a significant increase in the number of developing follicles in cultured rat ovaries treated with BMP-4 compared to the control. Similar to the Kit ligand, BMP-4 can act as a mitogen for granulosa cells and surrounding stromal cells, which can promote follicular transformation from primordial to further stages of development (Nilsson and Skinner, 2003). Indeed, our previous findings showed that the Ki67 and PCNA proliferation index of granulosa cells in transitional follicles was significantly higher in the HMB-exposed ovaries than in the control ones (Hułas-Stasiak et al., 2019). In addition, the present study indicated that the level of the Kit ligand and BMP-4 proteins within the piglets' ovaries significantly increased after the HMB treatment. Therefore, we speculate that HMB may enhance the expression of the Kit ligand and BMP-4 factors which appear to be responsible for accelerated follicular recruitment.

Basic FGF is another factor that has been identified to regulate the primordial to primary follicle transition (Nilsson and Skinner, 2004; Nilsson et al., 2001; Lu et al., 2015; Wang et al., 2014). In the present study, bFGF was detected in the oocytes and granulosa cells of primordial and developing follicles in both examined groups of piglets. This finding is consistent with many other studies describing the same localization in rat (Nilsson and Skinner, 2004; Nilsson et al., 2001), bovine (van Wezel et al., 1995), primate (Lu et al., 2015), and human (Wang et al., 2014; Yamamoto et al., 1997) ovaries. In vitro studies have shown that bFGF can enhance primordial follicle activation and growth to the preantral stage in various species (Lu et al., 2015; Nilsson and Skinner, 2004) including humans (Quennell et al., 2004; Wang et al., 2014) by stimulating the growth of theca and stromal cells (Nilsson and Skinner, 2004; Roberts and Ellis, 1999), as well as granulosa cell proliferation (Gospodarowicz and Bialecki, 1979; Lavranos et al., 1994; Roberts and Ellis, 1999). On the other hand, Ortega et al. (1998) reported that basic FGF is not absolutely required for primordial transition to occur, as bFGF knock-out mice are fertile. Surprisingly, in our study, the expression of bFGF protein decreased dramatically after the HMB treatment in relation to the control. It seems that the Kit ligand and BMP-4 or other growth factors may compensate for the low level of bFGF expression in HMB-treated ovaries.

IGF-1 has been shown in experimental animals to be a mitogen, a survival factor, and a stimulator of follicle growth (Martins et al., 2010; Monniaux et al., 1992; Stubbs et al., 2013; Zhao et al., 2001). Interestingly, in mice with a null mutation in the IGF-1 gene, follicle development is normal, until the late preantral stage, where development arrest is noted (Baker et al., 1996). Thus, IGF-1 appears to be important in the later stages of follicle development, but is not necessary at the initial stages 
of follicle growth associated with the primordial to primary transition (Baker et al., 1996). Kezele et al. (2002) also demonstrated that IGF-1 has a negligible effect on primordial to primary transition in the rat ovary. In the current study, IGF-1 and its receptor IGFR-1 were detected in the oocytes and granulosa cells of ovarian follicles. This pattern of IGF1/IGFR-1 cellular distribution is consistent with other studies (Martins et al., 2010; Poljicanin et al., 2015). Furthermore, we showed that the prenatal treatment with HMB resulted in diminished expression of IGF-1 and elevated expression of IGFR-1, compared to the control ovaries. Thus, we speculate that the insufficient level of IGF-1 proteins can impair IGF-1/IGFR-1 signalling, which may rather reflect its effect on egg nests apoptosis rather than on follicle transition.

\section{Conclusion}

Interactions between various factors such as the Kit ligand, BMP-4, bFGF, and IGF1/IGF-1R will provide a potential sequence of events involved in the initial process of folliculogenesis. The current findings show that BMP-4 and the Kit ligand can act as key regulators promoting follicle transition from the primordial to primary and transitional stages. On the other hand, these proteins may also control the oocyte apoptosis within egg nests. Furthermore, downregulation of the bFGF expression and altered IGF-1/IGFR-1 signalling can be involved in oocyte death and lead to reduction of the primordial follicle reserve. These data suggest that HMB can impair early folliculogenesis by modulating the expression of growth factors. The depletion of germ cell stock and acceleration of follicular recruitment can cause premature gonadal failure. The results obtained undermine the opinions about the safe impact of HMB on the body. Further research is necessary to gain information whether the changes observed in the neonatal period are reversible or they persist and lead to fertility problems during female reproductive life.

\section{References}

A a ron J.W., H s u e h A.J.W., K a w a m u r a K., C h e n g Y., F a u s e r B.C.J.M. (2015). Intraovarian control of early folliculogenesis. Endocr. Rev., 36: 1-24.

Baker J., Hardy M.P., Zhou J., Bondy C., Lupu F., Bellvé A.R., Efstratiadis A. (1996). Effects of an IGF-I gene null mutation on mouse reproduction. Mol. Endocrinol., 10: 903-918.

B e h 1 R., K a u 1 R. (2002). Insulin like growth factor 1 and regulation of ovarian function in mammals. Indian J. Exp. Biol., 40: 25-30.

B i la ńska-Osuchowska Z. (2006). Oogenesis in pig ovaries during the prenatal period: ultrastructure and morphometry. Reprod. Biol., 6: 161-193.

Blicharski T., Tomaszewska, E., Dobrowolski P., Hułas-Stasiak M., Muszyński S. (2017). A metabolite of leucine ( $\beta$-hydroxy- $\beta$-methylbutyrate) given to sows during pregnancy alters bone development of their newborn offspring by hormonal modulation. PLoS One, 12, e0179693.

B radford M.M. (1976). A rapid and sensitive method for quantification of microgram quantities of protein utilizing the principle of protein-die binding. Anal. Biochem., 72: 248-254.

Chan K.A., Ts oul is M.W., S loboda D.M. (2015). Early-life nutritional effects on the female reproductive system. J. Endocrinol., 224: R45-R62.

Child s A.J., Kinnell H.L., Collin s C.S., Hog g K.R., B a yne A.L., Green S.J., M c N e il - 
ly A.S., A n d e r s on R.A. (2010). BMP signaling in the human fetal ovary is developmentally regulated and promotes primordial germ cell apoptosis. Stem Cells, 28: 1368-1378.

C i eśl a k D., N i e r a d k a - I w a n i c k a B. (2018). $\beta$-hydroxy- $\beta$-methylbutyrate (HMB) supplementation during pregnancy and perinatal period in animals studies and possible application in humans. J. Educ. Health Sport, 8: 11-18.

C u nh a E.V. da, d e S o u za G.B., P a s s o s J.R.S., S i lv a A.W.B., D a u A.M., S a ra iv a M.V.A., L o b o R.N.B., S i 1 v a J.R.V. (2017). Effects of bone morphogenetic protein 4 (BMP4) on in vitro development and survival of bovine preantral follicles enclosed in fragments ovarian tissue. Zygote, 25: 256-264.

D on ed a L., K 1 ing er F.G., L a rizza L., D e Fel i c i M. (2002). KL/KIT co-expression in mouse fetal oocytes. Int. J. Dev. Biol., 46: 1015-1021.

Driancourt M.A., Reynaud K., Cortvrindt R., Smitz J. (2000). Roles of KIT and KIT LIGAND in ovarian function. Rev. Reprod., 5: 143-152.

Dupont C., Cordier A.G., Junien C., Mandon-Pépin B., Levy R., Chavatte-Pal$\mathrm{m}$ e $\mathrm{r}$ P. (2012). Maternal environment and the reproductive function of the offspring. Theriogenology, 78: 1405-1414.

Evans A.C., Moss a F., Wa $1 \mathrm{sh}$ S.W., S cheetz D., Jimenez-Kras sel F., Ire 1 and J.L., S mith G.W., Ireland J.J. (2012). Effects of maternal environment during gestation on ovarian folliculogenesis and consequences for fertility in bovine offspring. Reprod. Domest. Anim., 47 Suppl 4: 31-37.

Flu m m e r C., K risten se n N.B., The il P.K. (2012). Body composition of piglets from sows fed the leucine metabolite $\beta$-hydroxy- $\beta$-methylbutyrate in late gestation. J. Anim. Sci., 90: 442-444.

Gospodarowicz D., Bialecki H. (1979). Fibroblast and epidermal growth factors are mitogenic agents for cultured granulosa cells of rodent, porcine, and human origin. Endocrinology, 104: $757-764$.

Hø y e r P.E., B y s k o v A.G., Møllg år d K. (2005). Stem cell factor and c-Kit in human primordial germ cells and fetal ovaries. Mol. Cell Endocrinol., 234: 1-10.

Hułas-Stasiak M., Jakubowicz-Gil J., Dobrowolski P., Tomaszewska E., Muszyńs ki S. (2019). Maternal $\beta$-hydroxy- $\beta$-methylbutyrate (HMB) supplementation during pregnancy affects early folliculogenesis in the ovary of newborn piglets. Theriogenology, 128: 91-100.

H u s s e in M.R. (2005). Apoptosis in the ovary: molecular mechanisms. Hum. Reprod., 11: 162-178.

H u t K.J., M c L a u g h 1 in E.A., H o 11 a n d M.K. (2006). Kit ligand and c-Kit have diverse roles during mammalian oogenesis and folliculogenesis. Mol. Hum. Reprod., 12: 61-69.

J in X., H a n C.S., Yu F.Q., W e i P., H u Z.Y., L i u Y.X. (2004). Anti-apoptotic action of stem cell factor on oocytes in primordial follicles and its signal transduction. Mol. Reprod. Dev., 70: 82-90.

K a n g J.S., L e e C.J., L e e J.M., R h a J.Y., S o n g K.W., P ark M.H. (2003). Follicular expression of c-Kit/SCF and inhibin-alpha in mouse ovary during development. J. Histochem. Cytochem., 51: $1447-1458$.

Ke zele P.R., N ils s on E.E., S kin ner M.K. (2002). Insulin but not insulin-like growth factor-1 promotes the primordial to primary follicle transition. Mol. Cell. Endocrinol., 192: 37-43.

Krawczyk A., Rycerz K., Jaworska-Adamu J., Tomaszewska E., Dobrowolsk i P. (2016). Calretinin expression in hippocampus of mouse offspring from dams treated with $\beta$-hydroxy- $\beta$-methylbutyrate. Med. Weter., 72: 423-429.

Lavranos T.C., Rodger s H.F., B ert on c e 11 o I., R od ger s R.J. (1994). Anchorage-independent culture of bovine granulosa cells: The effects of basic fibroblast growth factor and dibutyryl cAMP on cell division and differentiation. Exp. Cell Res., 211: 245-251.

L u C.L., Yan J., Zhi X., X i a X., Wang T.R., Yan L.Y., Yu Y., Ding T., Ga o J.M., Li R., Q i a o J. (2015). Basic fibroblast growth factor promotes macaque follicle development in vitro. Reproduction, 149: 425-433.

Martins F.S., Saraiva M.V.A., Celestino J.J.H., Bruno J.B., Almeida A.P., Cunha R.M.S., S ilva J.R.V., Campello C.C., Lucci C.M., Matos M.H.T., Figueired o J.R. (2010). Expression of protein and mRNA encoding insulin growth factor-I (IGF-I) in goat ovarian follicles and the influence of IGF-I on in vitro development and survival of caprine preantral follicles. Anim. Reprod., 7: 349-361. 
Monniaux D., P is s e le t C. (1992). Control of proliferation and differentiation of ovine granulosa cells by insulin-like growth factor-I and follicle-stimulating hormone in vitro. Biol. Reprod., 46: 109-111.

M orit a Y., Tilly J.L. (1999). Oocyte apoptosis: like sand through an hourglass. Dev. Biol., 213: $1-17$.

Morita Y., Manganaro T.F., Ta o X.J., Martimbeau S., D on aho e P.K., Tilly J.L. (1999). Requirement for phosphatidylinositol-3'-kinase in cytokine-mediated germ cell survival during fetal oogenesis in the mouse. Endocrinology, 140: 941-949.

Nils s on E.E., Skinner M.K. (2003). Bone morphogenetic protein-4 acts as an ovarian follicle survival factor and promotes primordial follicle development. Biol. Reprod., 69: 1265-1272.

Nils s on E.E., Skinner M.K. (2004). Kit ligand and basic fibroblast growth factor interactions in the induction of ovarian primordial to primary follicle transition. Mol. Cell. Endocrinol., 214: $19-25$.

Nils s o n E., P a r r o t t J.A., S k in n e r M.K. (2001). Basic fibroblast growth factor induces primordial follicle development and initiates folliculogenesis. Mol. Cell. Endocrinol., 175: 123-130.

Nils s o n E.E., K e z e le P., S k in ner M.K. (2002). Leukemia inhibitory factor (LIF) promotes the primordial to primary follicle transition in rat ovaries. Mol. Cell. Endocrinol., 188: 65-73.

NRC, National Research Council (2012). Nutrient Requirements of Swine. National Academy Press, Washington, DC, USA, 11th ed., 420 pp.

Ortega S., Ittmann M., Tsang S.H., Erlich M., Basilico C. (1998). Neuronal defects and wound healing in mice lacking fibroblast growth factor 2. Proc. Natl. Acad. Sci., USA 95: 5672-5677.

P a r r o t J.A., S k i n n e r M.K., (1999). Kit ligand/stem cell factor induces primordial follicle development and initiates folliculogenesis. Endocrinology, 140: 4262-4271.

P edersen T., P e ters H. (1968). Proposal for a classification of oocytes and follicles in the mouse ovary. Reproduction, 17: 555-557.

Poljicanin A., Filipovic N., Vukusic Pusic T., Soljic V., Caric A., Saraga-Bab i c M., Vu k oj e vi c K. (2015). Expression pattern of RAGE and IGF-1 in the human fetal ovary and ovarian serous carcinoma. Acta Histochem., 117: 468-476.

Qu en ne 11 J.H., S t a n t on J.A.L., H u r s t P.R. (2004). Basic fibroblast growth factor expression in isolated small human ovarian follicles. Mol. Hum. Reprod., 10: 623-628.

Res n i ck J.L., Ortiz M., K e 11 e r J.R., D o n ov a n P.J. (1998). Role of fibroblast growth factors and their receptors in mouse primordial germ cell growth. Biol. Reprod., 59: 1224-1229.

Reynaud K., Cortvrindt R., Smitz J., Driancourt M.A. (2000). Effects of Kit Ligand and anti-Kit antibody on growth of cultured mouse preantral follicles. Mol. Reprod. Dev., 56: 483-494.

R o b e r t s R.D., E 11 i s R.C.L. (1999). Mitogenic effects of fibroblast growth factors on chicken granulosa cell and theca cells in vitro. Biol. Reprod., 61: 1387-1392.

Ros s A.J., Tilman C., Yao H., Mac Laughlin D., Capela B. (2003). AMH induces mesonephric cell migration in XX gonads. Mol. Cell. Endocrinol., 211: 1-7.

Shim izu T., Yok o o M., Mi y a ke Y., S a s a d a H., S a to E. (2004). Differential expression of bone morphogenetic protein 4-6 (BMP-4,5 and 6) and growth differentiation factor-9 (GDF-9) during ovarian development in neonatal pigs. Domest. Anim. Enocrinol., 27: 397-405.

S tu b bs S.A., We bber L.J., S t a rk J., R i c e S., Margara R., L a very S., Trew G.H., Hardy K., Franks S. (2013). Role of insulin-like growth factors in initiation of follicle growth in normal and polycystic human ovaries. J. Clin. Endocrinol. Metab., 98: 3298-3305.

Świetlicka I., Muszyński S., Tomaszewska E., Dobrowolski P., Kwaśniews k a A., Ś w i e tli c k i M., S k i c A., G oła c ki K. (2016). Prenatally administered HMB modifies the enamel surface roughness in spiny mice offspring: An atomic force microscopy study. Arch. Oral Biol., 70: 24-31.

T a t a r a M.R., Śl i w a E., K r u p s k i W. (2007). Prenatal programming of skeletal development in the offspring: effects of maternal treatment with $\beta$-hydroxy- $\beta$-methylbutyrate (HMB) on femur properties in pigs at slaughter age. Bone, 40: 1615-1622.

T in g e n C., K i m A., W o o d r u f T.K. (2009). The primordial pool of follicles and nest breakdown in mammalian ovaries. Mol. Hum. Reprod., 15: 795-803. 
Wan H.F., Zhu J.T., Shen Y., Xiang X., Yin H.J., Fang Z.F., Che L.Q., Lin Y., Xu S.Y., F eng B., W u D. (2016 a). Effects of dietary supplementation of $\beta$-hydroxy- $\beta$-methylbutyrate on sow performance and mRNA expression of myogenic markers in skeletal muscle of neonatal piglets. Reprod. Domest. Anim., 51: 134-142.

Wan H., Zhu J., Su G., Li u Y., Hu a L., Hu L., Wu C., Zhang R., Zhou P., Shen Y., Lin Y., X u S., F ang Z., Ch e L., F eng B., Wu D. (2016 b). Dietary supplementation with $\beta$-hydroxy$\beta$-methylbutyrate calcium during the early postnatal period accelerates skeletal muscle fiber growth and maturity in intra-uterine growth-retarded and normal-birth-weight piglets. Br. J. Nutr., 115: 1360-1309.

Wan H., Zhu J., Wu C., Zhou P., Shen Y., Lin Y., Xu S., Che L., F eng B., Li J., F ang Z., W u D. (2017). Transfer of $\beta$-hydroxy- $\beta$-methylbutyrate from sows to their offspring and its impact on muscle fibre type transformation and performance in pigs. J. Anim. Sci. Biotechnol., 8: 2.

Wang T., Yan L., Yan J., Lu C., Xi a X., Y in T.L., Z hu X.H., G a o J.M., D ing T., Hu W.H., Gu o H.Y., Li R., Q i a o J. (2014). Basic fibroblast growth factor promotes the development of human ovarian early follicle during growth in vitro. Hum. Reprod., 29: 568-576.

We ze 1 I.L. van, U map a thy siva m K., Ti 11 e y W.D., R od gers R.J. (1995). Immunohistochemical localization of basic fibroblast growth factor in bovine ovarian follicles. Mol. Cell. Endocrinol., 115: 133-140.

Wils on G.J., Wils on J.M., M a n n in e n A.H. (2008). Effects of beta-hydroxy-beta-methylbutyrate $(\mathrm{HMB})$ on exercise performance and body composition across varying levels of age, sex, and training experience: a review. Nutr. Metab., 5: 1.

Wils on J.M., Fits chen P.J., Campbell B., Wils on G.J., Zanchi N., Taylor L., Wil b orn C., K a $1 \mathrm{~m}$ a n D.S., S to u t J.R., Ho ffman J.R., Z i e gen fus s T.N., L o p e z H.L., K re ider R.B., S m ith-R u an A.E., A n t on i o J. (2013). International Society of Sports Nutrition Position Stand: beta-hydroxy-beta-methylbutyrate (HMB). J. Int. Soc. Sports Nutr., 10: 6.

Yamamoto S., Konishi I., Nanbu K., Komats u T., Mandai M., Kuroda H., Matsus h it a K., M ori T. (1997). Immunohistochemical localization of basic fibroblast growth factor (bFGF) during folliculogenesis in the human ovary. Gynecol. Endocrinol., 11: 223-230.

Zhao J., Tavene M.A., Van Der Weijden G.C., Bevers M.M., Van Den Hurk R. (2001). Insulin-like growth factor-I (IGF-I) stimulates the development of cultured rat pre-antral follicles. Mol. Reprod. Develop., 58: 287-296.

Received: 16 IX 2019

Accepted: 25 II 2020 